\title{
Variability in multimodality treatment influences survival in non- metastatic gastric cancer patients
}

\author{
Tuyen Hoang ${ }^{1 \#}$, Farshid Dayyani ${ }^{2 \#}$, Ariceli Alfaro ${ }^{3}$, Jasmine Huynh ${ }^{4}$, Jingran $\mathrm{Ji}^{4}$, Andrew H. Ko ${ }^{3}$, \\ May $\mathrm{Cho}^{5}$, Darryl Hiyama ${ }^{6}$ \\ ${ }^{1}$ Institute for Clinical and Translational Science, ${ }^{2}$ Division of Hematology and Oncology, Department of Medicine, University of California in Irvine, \\ Irvine, USA; ${ }^{3}$ Gastrointestinal Oncology Program, University of California in San Francisco, San Francisco, USA; ${ }^{4}$ School of Medicine, ${ }^{5}$ Division of \\ Hematology and Oncology, Department of Medicine, University of California in Davis, Davis, USA; ${ }^{6}$ Department of General Surgery, University of \\ California in Los Angeles, Los Angeles, USA \\ Contributions: (I) Conception and design: T Hoang, F Dayyani, D Hiyama; (II) Administrative support: F Dayyani, D Hiyama; (III) Provision of study \\ materials or patients: F Dayyani, D Hiyama, AH Ko, M Cho; (IV) Collection and assembly of data: T Hoang, A Alfaro, J Huynh, J Ji; (V) Data analysis \\ and interpretation: T Hoang, F Dayyani, D Hiyama, AH Ko; (VI) Manuscript writing: All authors; (VII) Final approval of manuscript: All authors. \\ \#These authors contributed equally to this work. \\ Correspondence to: Tuyen Hoang, PhD. 843 Health Sciences Road, Irvine, CA 92697, USA. Email: tuyenh1@uci.edu.
}

Background: While gastric cancer is a leading cause of cancer-related mortality in Eastern Europe and Asia, it is less common in the USA. Recommendations regarding optimal treatment of non-metastatic gastric cancer (nmGC) with regard to type and extent of surgery, choice and sequence of chemotherapeutic agents, and use of radiation therapy vary across geographic locations. To determine how variability in treatment practices affects patient outcomes, we conducted a retrospective study to evaluate clinical outcomes in nmGC patients treated at four high-volume academic institutions.

Methods: California Cancer Registry data were collected for nmGC patients who underwent gastrectomy with curative intent from 2010 to 2018. We conducted chart reviews of the patients' electronic health records to validate clinical factors and outcomes. We performed multivariable Cox regressions to determine prognostic factors for outcomes.

Results: Demographics of study cohort ( $n=326)$ : mean age 66 years; $64 \%$ male; $44 \%$ Caucasian, 35\% Asian, 16\% Latino. Tumor stage: $48 \%$ loco-regional (pT4 or pN1+) vs. 52\% localized (pT1-3, pN0). Histology: $47 \%$ intestinal, $30 \%$ diffuse, $8 \%$ mixed, $15 \%$ unknown. Surgery: $34 \%$ open gastrectomy, $48 \%$ laparoscopic, $18 \%$ unknown; number of recovered lymph nodes varied from 0 to 60 in any tumor stage. Chemotherapy: $20 \%$ neoadjuvant alone, $25 \%$ adjuvant alone, $16 \%$ perioperative, $39 \%$ none. Multimodality therapy: $44 \%$ surgery only, $31 \%$ chemotherapy, $25 \%$ chemotherapy and radiation. With a median post-surgical follow-up of 6 years, $24 \%$ of patients developed recurrence and $40 \%$ had died. Compared to open surgery, laparoscopic surgeries were associated with fewer recovered lymph nodes (mean $=18$ vs. $25, \mathrm{P}=0.0042$ ). Fewer recovered lymph nodes were associated with a significant decrease in 5 -year overall survival [hazard ratio $(\mathrm{HR})=1.9$, 95\% confidence interval (CI): 1.3-2.8]. Timing of chemotherapy and addition of radiation therapy to chemotherapy did not confer further improvements in survival; in contrast, greater lymph node recovery plus chemotherapy were associated with a significant increase in survival ( $\mathrm{HR}=0.3,95 \% \mathrm{CI}$ : 0.1-0.6)

Conclusions: This study highlights major practice differences in the management of nmGC patients across providers and institutions. Further efforts should be made to standardize the use of chemotherapy and adequate recovery and assessment of lymph nodes in this patient population.

Keywords: Gastric; cancer; multimodality; treatment; survival

Submitted May 11, 2020. Accepted for publication Sep 04, 2020.

doi: 10.21037/jgo-20-212

View this article at: http://dx.doi.org/10.21037/jgo-20-212 


\section{Introduction}

Gastric cancer is the fifth most common malignancy and the third leading cause of cancer mortality worldwide (1). While gastric cancer is a leading cause of cancer-related mortality in Eastern Europe and Asia, it is less common in the United States. Recommendations regarding optimal treatment of non-metastatic gastric cancer with regard to type and extent of surgery, choice and sequence of chemotherapeutic agents, and use of radiation therapy vary depending on geographic location. In the United States, based on the INT0116 trial (2), adjuvant fluoropyrimidinebased chemoradiation has been one commonly used approach. However, the quality of surgical resection in that trial has been debated and it is postulated that the addition of radiation compensated for the fact that most patients underwent a D1 resection. The standard for perioperative chemotherapy without radiation was based on the MAGIC trial which compared ECF (epirubicin, cisplatin, 5-FU) given before and after surgery with surgery alone and demonstrated an improvement in 5-year survival from $23 \%$ to $36 \%$ (3). The FNCLCC and FFCD trial reported similar results with the use of a 28-day regimen of perioperative cisplatin and 5-FU (4). More recently, the German FLOTAIO trial showed superiority of peri-operative FLOT (5FU, leucovorin, oxaliplatin, docetaxel) over ECF (5). As a result, perioperative chemotherapy has been widely adopted as the standard of care throughout most of the UK and Europe. Meanwhile, throughout Asia, most patients are treated with D2 gastrectomy followed by adjuvant chemotherapy alone based on the CLASSIC and ACT-GC trials $(6,7)$.

In addition, there is heterogeneity and lack of consensus regarding the optimal extent for lymph node dissection during gastrectomy $(8,9)$. In eastern Asia, gastrectomy with D2 lymphadenectomy is the standard treatment for curable gastric cancer while surgical practice in western Europe and the USA are guided by the UK Medical Research Council trial (10) and the Dutch Gastric Cancer trial (11). The 5-year follow-up data from these two large European trials showed that extended D2 lymphadenectomy did not improve survival and was associated with significantly higher morbidity and mortality compared with limited D1 lymphadenectomy. The higher morbidity and mortality rates were mostly associated with pancreatico-splenectomy, which was an integral part of the D2 resection in both trials. Recent clinical trials have confirmed that pancreaspreserving D2 resection yield better survival outcomes than
D1 resection, but more extended D3 resection does not appear to be better than D2 resection (12-15).

To determine how variability in treatment practices affects patient outcomes, we conducted a retrospective study in patients with non-metastatic gastric cancer treated at four University of California (UC) medical centers. The authors present the study in accordance with the STROBE reporting checklist. Available at http://dx.doi.org/10.21037/ jgo-20-212.

\section{Methods}

\section{Study cobort}

The study was conducted in accordance with the Declaration of Helsinki (as revised in 2013). This study was approved by the Institutional Review Boards at the Universities of California in Davis, Irvine, Los Angeles, and San Francisco prior to the collection of data (UC Reliance No. 2843). Because of the retrospective nature of the research, the requirement for informed consent was waived. The study cohort which was retrospectively identified from electronic databases included patients diagnosed with non-metastatic gastric adenocarcinoma who underwent gastrectomy with curative intent at four medical centers from 2010 to 2018. Patients receiving endoscopic surgery were not included in this study because endoscopic therapy for early gastric adenocarcinoma was limited in frequency at the study institutions and often reserved for very early tumors (Tis) or for patients unable to undergo formal resection.

\section{Data collection}

We gathered retrospective data from UC electronic health records and California Cancer Registry databases. UC electronic health data were obtained through chart reviews, including patient demographics (age, sex, race), risk factors (smoking and alcohol use, significant weight loss, history of cancer, comorbidities), tumor characteristics (stage based on the AJCC $7^{\text {th }}$ edition, histology, tumor size), surgical characteristics (laparoscopic technology, partial or total gastrectomy, number of recovered lymph nodes, and resection margin status), types of therapy (chemotherapy, radiation), administration of chemotherapy (neoadjuvant alone, adjuvant alone, and perioperative), and outcomes (cancer recurrence and death). The cutoffs of these variables were chosen as they were mentioned in literature guidelines 
for lymph node dissection (16). UC electronic health data and California Cancer Registry data were linked by patient identifiers in order to obtain more complete and accurate information about patients' treatment and follow-up.

\section{Statistical analyses}

We summarized the demographic, clinical, tumor, and treatment characteristics of the study patients using descriptive statistics such as percentage, mean, and standard deviation. Then, we summarized the outcomes including cancer recurrence events and death events using percentages. We compared these variables across the four institutions using $t$-tests and chi-square tests whenever applicable.

To determine patient characteristics associated with higher risk of cancer recurrence and death, we performed a disease-free analysis and an overall survival analysis. Recurrence events were used for the disease-free analysis and death events of any cause were used for the overall survival analysis. In these analyses, we used a multivariable Cox regression model with proportional hazard distribution. The unit of analysis was a patient. The dependent variable was the time from gastrectomy to the first cancer recurrence (or death of any cause). For patients who had never had recurrence (or death) or were lost to follow-up, the time was censored by their last visit. The independent variables included patient demographic, clinical, and tumor variables. The results of these analyses included hazard ratios (HRs) which were used to determine the patient characteristics associated with higher risk of cancer recurrence and death. A HR of value greater than 1 indicated that one group of patients was at greater risk of recurrence (or death) than the other group; a HR of value less than 1 indicated lower risk; and a HR of value equal to 1 indicated equal risk. All HRs obtained from these analyses were adjusted for demographic, clinical, tumor, and treatment variables.

To evaluate the effect of lymph node recovery on survival, we first determined the median number of lymph nodes in each tumor stage. We flagged each patient whether his/her number of recovered lymph nodes was above or below stage-specific median value. Then we performed a Cox regression analysis to compare overall survival between patients with lymph node recovery above $v s$. below the median. All results obtained from this analysis were adjusted for the demographic, clinical, tumor, and treatment variables that had been found significantly associated with survival in the preceding analysis. Lastly, we repeated this analysis using various thresholds other than the median values to determine what thresholds of lymph node recovery would yield optimal survival.

To compare survival among multimodality treatments, we classified patients into subgroups by tumor stage, extent of lymph node recovery, and receipt of chemotherapy. Then we evaluated whether the subgroups were clinically similar by comparing Charlson comorbidity score and weight loss status at diagnosis among the subgroups using chi-square tests. According to the cancer registry data dictionary (17), the Charlson comorbidity score is a weighted index based on the presence of certain comorbid conditions twelve months prior through 6 months following the cancer diagnosis (as determined through ICD-9 and ICD-10 diagnosis codes), weighted by the severity of those conditions. It is intended to be a measure of burden of serious comorbidity, not the number of comorbidities. The following conditions were included in this index: congestive heart failure, myocardial infarction (acute and old), peripheral vascular disease, cerebrovascular disease, dementia, chronic pulmonary disease, rheumatologic disease, peptic ulcer disease, mild liver disease, moderate/severe liver disease, diabetes, diabetes with chronic complications, hemi- or paraplegia, renal disease, and acquired immune deficiency syndrome. In the end, we performed a multivariable Cox regression analysis to compare overall survival among the subgroups, adjusting for the demographic, clinical, tumor, and treatment variables that had been found significantly associated with survival in the previous analysis.

\section{Results}

Patient demographic, clinical, tumor, and treatment characteristics are summarized in Table 1.

\section{Demographics}

A total of 326 patients fulfilling the study inclusion criteria were included in the analysis. Age at diagnosis ranged from 24 to 98 years, with a mean of 66 years $(S D=12)$. About $64 \%$ of the patients were male and $36 \%$ were female. The most common ethnicity was Caucasian (44\%), followed by Asian (35\%) and Hispanic (16\%). Age and race differed significantly across institutions $(\mathrm{P}<0.0040)$.

\section{Clinical characteristics}

About $52 \%$ of the patients smoked cigarettes or consumed 
Table 1 Demographic, clinical, tumor, and treatment characteristics of the study patients

\begin{tabular}{|c|c|c|c|c|c|c|}
\hline Characteristics & \multicolumn{5}{|c|}{ Institutions } & $P$ value \\
\hline \multicolumn{7}{|l|}{ Demographics } \\
\hline Age at diagnosis (years) & & & & & & 0.0035 \\
\hline Mean & 65.8 & 66.8 & 58.1 & 67.1 & 65.8 & \\
\hline Range & $26-88$ & 24-92 & $25-80$ & $39-98$ & $24-98$ & \\
\hline $\operatorname{Sex}(\%)$ & & & & & & 0.2184 \\
\hline Male & 63.9 & 66.9 & 46.7 & 65.0 & 63.8 & \\
\hline Female & 36.1 & 33.1 & 53.3 & 35.0 & 36.2 & \\
\hline Asian & 48.5 & 25.2 & 13.3 & 48.3 & 35.3 & \\
\hline Hispanic & 18.5 & 15.1 & 20.0 & 11.7 & 16.0 & \\
\hline African American & 0 & 2.1 & 3.4 & 5.0 & 2.1 & \\
\hline Others & 0 & 2.9 & 10.0 & 1.7 & 2.4 & \\
\hline \multicolumn{7}{|l|}{ Clinical characteristics } \\
\hline Smoking/alcohol (\%) & & & & & & $<0.0001$ \\
\hline Yes & 41.2 & 69.1 & 70.0 & 21.7 & 52.2 & \\
\hline No or unknown & 58.8 & 30.9 & 30.0 & 78.3 & 47.8 & \\
\hline No or unknown & 90.7 & 83.5 & 73.3 & 95.0 & 86.8 & \\
\hline Charlson comorbidity (\%) & & & & & & 0.5941 \\
\hline Low burden (score <3) & 91.7 & 86.3 & 86.7 & 90.0 & 88.7 & \\
\hline High burden (score $=3+$ ) & 8.3 & 13.7 & 13.3 & 10.0 & 11.3 & \\
\hline \multicolumn{7}{|l|}{ Tumor characteristics } \\
\hline pT stage (\%) & & & & & & 0.0005 \\
\hline 1 & 44.3 & 33.1 & 16.7 & 28.3 & 34.0 & \\
\hline 2 & 13.4 & 20.1 & 6.7 & 13.3 & 15.6 & \\
\hline 3 & 29.9 & 35.3 & 43.3 & 25.0 & 32.5 & \\
\hline 4 & 12.4 & 11.5 & 33.3 & 33.4 & 17.9 & \\
\hline
\end{tabular}

Table 1 (continued) 
Table 1 (continued)

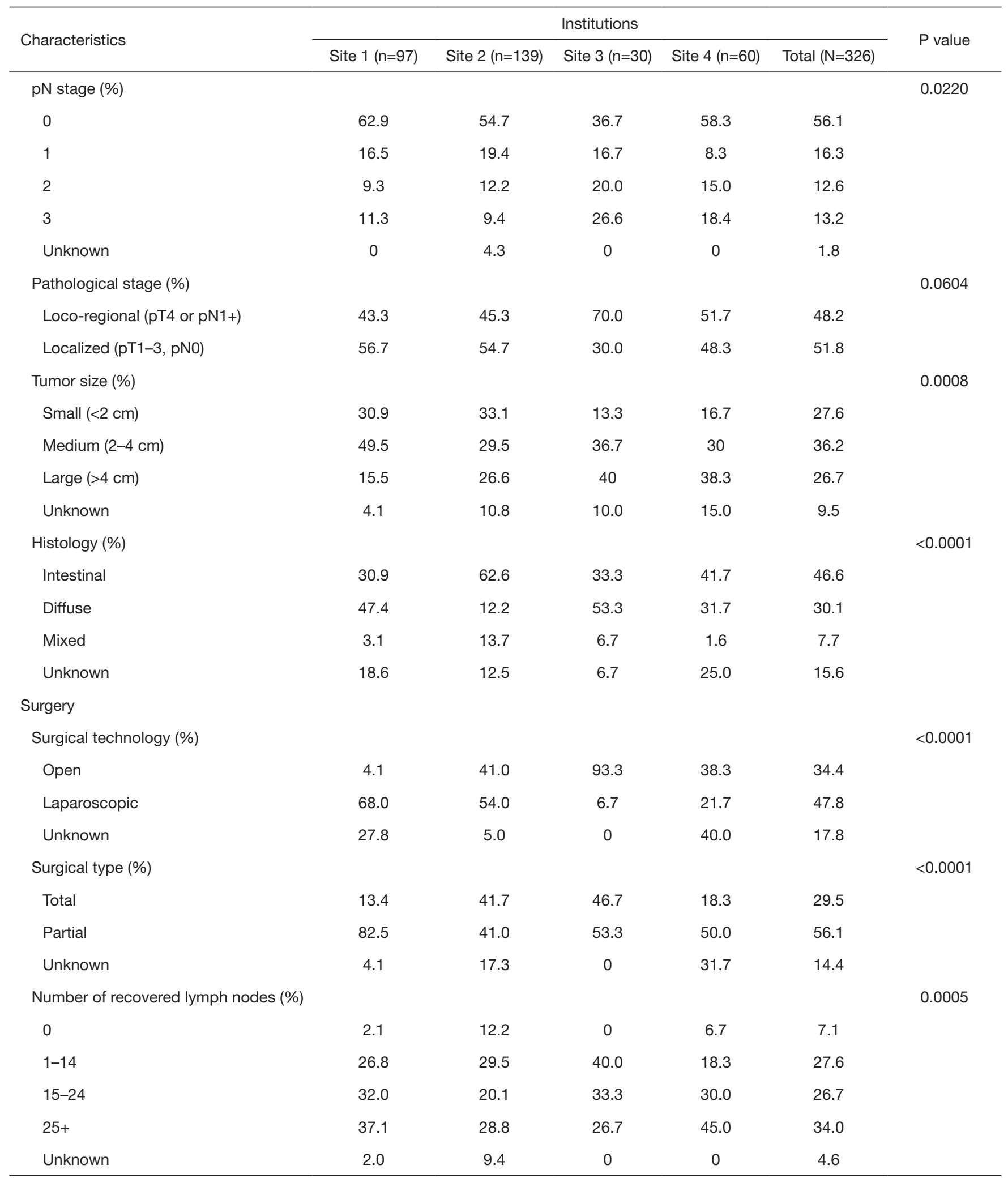

Table 1 (continued) 
Table 1 (continued)

\begin{tabular}{|c|c|c|c|c|c|c|}
\hline Characteristics & \multicolumn{5}{|c|}{ Institutions } & $P$ value \\
\hline Positive resection margins (\%) & & & & & & 0.0002 \\
\hline Yes & 8.3 & 5.0 & 30.0 & 6.7 & 8.6 & \\
\hline No & 91.7 & 95.0 & 70.0 & 93.3 & 91.4 & \\
\hline \multicolumn{7}{|l|}{ Chemotherapy } \\
\hline Chemotherapy administration (\%) & & & & & & 0.1180 \\
\hline Neoadjuvant alone & 21.6 & 21.6 & 20.0 & 11.7 & 19.6 & \\
\hline Adjuvant alone & 17.5 & 25.9 & 33.3 & 33.3 & 25.5 & \\
\hline Perioperative (before \& after surgery) & 12.4 & 19.4 & 20.0 & 13.3 & 16.3 & \\
\hline Multimodality (\%) & & & & & & 0.1880 \\
\hline Surgery only & 48.5 & 45.3 & 30.0 & 41.7 & 44.2 & \\
\hline Surgery/chemotherapy & 24.7 & 28.1 & 40.0 & 41.7 & 30.7 & \\
\hline Surgery/chemotherapy/radiation & 26.8 & 26.6 & 30.0 & 16.6 & 25.1 & \\
\hline
\end{tabular}

alcohol on a regular basis; $36 \%$ experienced significant weight loss at the time of cancer diagnosis; and $13 \%$ had a history of cancer other than gastric cancer. About $11 \%$ of the patients had high comorbidity burden based on Charlson comorbidity score of 3 or greater. All clinical characteristics, except comorbidity, varied significantly among institutions $(\mathrm{P}<0.0500)$.

\section{Tumor characteristics}

There was an approximately even distribution between loco-regional (pT4 or pN1+) and localized (pT1-3, pN0) tumors, as well as across tumor size. In terms of histology, $47 \%$ of the tumors were diffuse type; $30 \%$ were intestinal type; $8 \%$ were mixed type; and $16 \%$ were unknown histology. Tumor size and histology were significantly different across institutions $(\mathrm{P}<0.0010)$.

\section{Surgery}

A majority of patients (48\%) underwent laparoscopic gastrectomy. Total gastrectomy was performed in $30 \%$.
Lymph node recovery varied widely: $35 \%$ of patients with fewer than 15 lymph nodes examined, 27\% with 15-24 lymph nodes, and $34 \%$ with $25+$ lymph nodes. Positive resection margins were seen in $9 \%$ of patients. All of these surgical characteristics varied significantly across institutions $(\mathrm{P}<0.0001)$.

\section{Chemotherapy administration}

About $20 \%$ of the patients received neoadjuvant chemotherapy alone; $25 \%$ received adjuvant chemotherapy alone; and $16 \%$ received perioperative chemotherapy (i.e., chemotherapy both before and after surgery). Chemotherapy records were not available in $39 \%$ of patients. Variation in chemotherapy administration across institutions was not statistically significant $(\mathrm{P}=0.1180)$.

\section{Multimodality treatment}

About $44 \%$ of the patients received surgery only; $31 \%$ received surgery and chemotherapy; and $25 \%$ received surgery, chemotherapy, and radiation. 
Table 2 Outcomes

\begin{tabular}{|c|c|c|c|c|c|c|}
\hline Outcomes & Site $1(n=97)$ & Site $2(n=139)$ & Site $3(n=30)$ & Site $4(n=60)$ & Total $(\mathrm{N}=326)$ & $P$ value \\
\hline Distant recurrence & 14.4 & 22.3 & 30 & 16.7 & 19.6 & \\
\hline Local recurrence & 3.1 & 5.0 & 3.3 & 6.7 & 4.6 & \\
\hline None or unknown & 82.5 & 72.7 & 66.7 & 76.6 & 75.8 & \\
\hline Dead & 24.7 & 47.5 & 56.7 & 38.3 & 39.9 & \\
\hline Alive & 75.3 & 52.5 & 43.3 & 61.7 & 60.1 & \\
\hline
\end{tabular}

${ }^{*}$, with a median follow-up of 6 years after gastrectomy.

\section{Patient characteristics associated with higher risk of cancer recurrence and death}

Clinical outcomes are summarized in Table 2. After a median post-surgical follow-up of 6 years, $24 \%$ of the patients had cancer recurrence and $40 \%$ had died of any cause. We found that the difference between the death events and the recurrence events were due to the fact that some patients were lost to follow-up and their deaths were not documented at the study institutions; although we were able to obtain all death events from the cancer registry database, we were not able to obtain the causes of death for patients who died outside of the study institutions. As shown in Table 3, the patient characteristics significantly associated with higher risk of cancer recurrence and death included weight loss at diagnosis [recurrence: $\mathrm{HR}=3.0$, 95\% confidence interval (CI): 2.0-4.7; death: $\mathrm{HR}=2.4$, 95\% CI: 1.7-3.3] and loco-regional disease (recurrence: HR $=3.0,95 \%$ CI: $1.9-4.7$; death: HR $=2.7,95 \%$ CI: $1.9-3.9)$. Specifically, 5 -year overall survival was $35 \%$ in patients with loco-regional tumors or weight loss as compared to $70 \%$ in those without these factors $(\mathrm{P}<0.0001)$. Although not associated with cancer recurrence, Caucasian patients had worse overall survival compared to Asian patients $(\mathrm{HR}=1.6$, 95\% CI: $1.1-2.5$ ).

As a note, when we compared overall survival by individual $\mathrm{T}$ and $\mathrm{N}$ stages, we found that survival decreased as $\mathrm{T}$ and $\mathrm{N}$ stages increased ( $\mathrm{T}$ stage: $\mathrm{HR}=1.7,95 \% \mathrm{CI}$ : 1.4-2.0; N stage: $\mathrm{HR}=1.3,95 \% \mathrm{CI}$ : $1.2-1.4$; see graphs in Figures S1,S2). However, when we compared survival among the subgroups of patients receiving different treatment modalities stratified by individual $\mathrm{T}$ and $\mathrm{N}$ stages, the sample size in each subgroup was too small to demonstrate statistical significance. Therefore, we decided to combine $\mathrm{T}$ and $\mathrm{N}$ stages into two categories (T1-3, N0) vs. (T4, N1+) to increase the sample sizes of the comparison groups; and we defined (T1-3, N0) tumors as localized disease and (T4, $\mathrm{N} 1+)$ tumors as loco-regional disease.

\section{Variability in multimodality treatments infuenced survival}

We observed great variability in surgery and therapy practices across providers and institutions (Table S1). Among patients with loco-regional tumors receiving chemotherapy, the timing of administration varied: $20 \%$ neoadjuvant alone, $43 \%$ adjuvant alone, and $22 \%$ perioperative. Additionally, among patients with loco-regional tumors, $23 \%$ received surgery alone, $42 \%$ received surgery and chemotherapy, and $35 \%$ received surgery, chemotherapy, and radiation. Furthermore, Figure 1 shows that lymph node recovery varied greatly within a given tumor stage and across tumor stages; the number of recovered lymph nodes ranged from 0 to 60 within any tumor stage, with the median value varying from 12 to 31 across tumor stages. Last but not least, we found that laparoscopic surgeries were associated with fewer recovered lymph nodes compared to open surgeries after adjusted for tumor stage (mean number of lymph nodes $=18$ vs. 25, $\mathrm{P}=0.0042$ ).

As shown in Figure 2, increase in 5-year overall survival due to higher lymph node recovery was most profound among patients with localized tumors (HR $=0.4,95 \%$ CI: $0.2-0.8$ ) while increase in 5-year overall survival due to chemotherapy was most profound among patients with loco-regional tumors (HR $=0.4,95 \% \mathrm{CI}$ : 0.2-0.8). Specifically, among patients with localized tumors, 5 -year overall survival increased from $60 \%$ among those who 
Table 3 Patient characteristics associated with higher risk of cancer recurrence and death

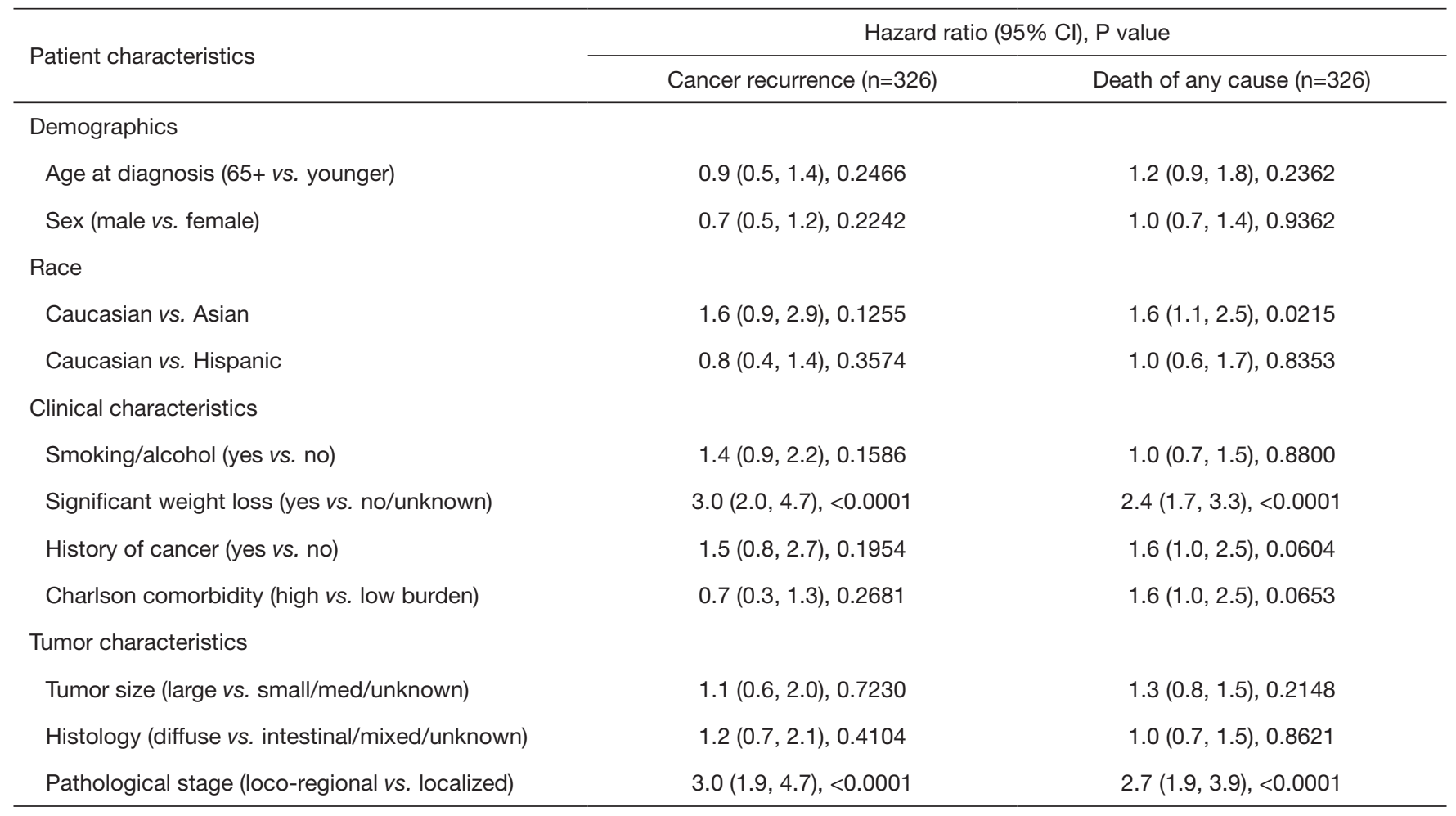

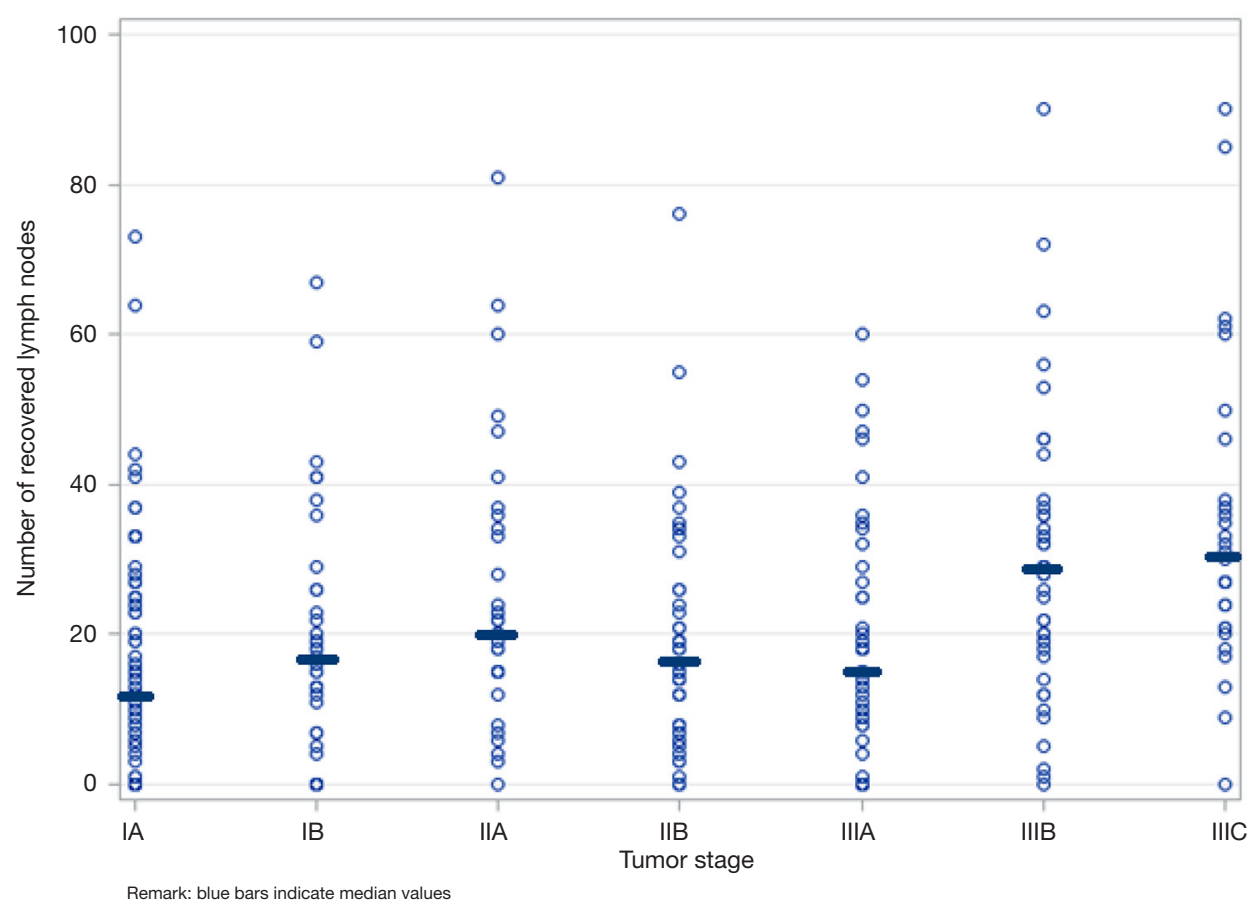

Figure 1 Variability of lymph node recovery within and across tumor stages. Blue bars indicate median values. 


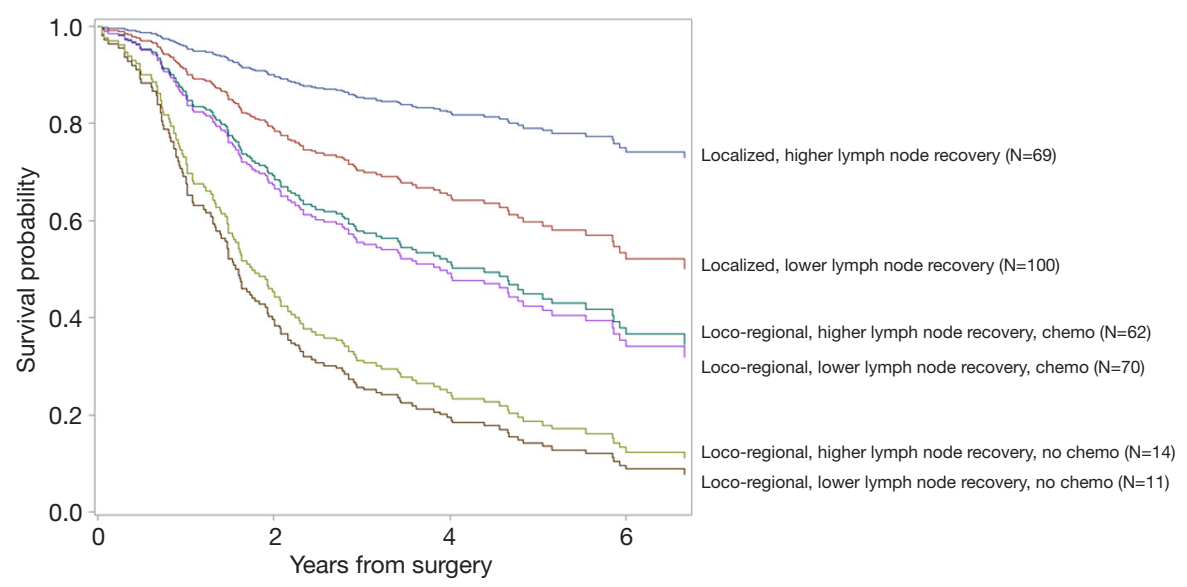

Figure 2 Comparison of overall survival among treatment groups, stratified by tumor stage.

received lower lymph node recovery to $80 \%$ among those who received higher lymph node recovery $(\mathrm{P}<0.0001)$. Among patients with loco-regional tumors, 5 -year overall survival increased from $20 \%$ among patients without chemotherapy to $45 \%$ among patients with chemotherapy $(\mathrm{P}<0.0001)$. Of note, the treatment subgroups were clinically comparable, with no statistically significant differences in the percentages of high comorbidity burden and weight loss at diagnosis (Table S2).

For patients with loco-regional tumors, timing of administration did not appear to be important; 5-year overall survival was not statistically different between adjuvant alone $v s$. perioperative chemotherapy $(\mathrm{HR}=0.9$, 95\% CI: 0.5-1.7, see figure in Figure S3) and adjuvant alone $v s$. neoadjuvant chemotherapy alone ( $\mathrm{HR}=0.7,95 \%$ CI: 0.4-1.2). Additionally, 5-year overall survival was not statistically significant between chemotherapy plus radiation vs. chemotherapy alone (HR $=0.9,95 \%$ CI: $0.5-1.5$, see figure in Figure S4).

\section{Discussion}

Literature shows great variability in gastric cancer treatments around the world. In this study, we determined whether variability in treatment practices affect survival in patients with non-metastatic gastric cancer treated with modern multimodality strategies at four high-volume academic institutions within the UC system.

Our study cohort was heterogeneous in demographic, clinical, and tumor characteristics including a wide range of predisposing risk factors such as regular cigarette smoking, alcohol consumption, history of cancer, and a full spectrum of disease severity in tumor stage, size, and histology. We found tumor stage and weight loss at diagnosis significantly associated with higher risk of cancer recurrence and death, with a 5 -year overall survival in patients with these factors to be half of that compared to patients without these factors. The prognostic significance of tumor stage in resected gastric cancer observed in this study are consistent with literature (18-20).

We observed great variability in surgical practices across providers and institutions. Our surgical data was derived from surgeries performed after 2010 when the well-known Dutch D1D2 trial confirmed the superior benefits of extended D2 lymphadenectomy over limited D1 lymphadenectomy on patient survival (11). Yet, we observed that the number of recovered lymph nodes in our study cohort varied from 0 to 60 in any tumor stage. When we examined the number of recovered lymph nodes at the provider level, we found no correlation between the number of recovered lymph nodes and tumor extent. However, in aggregation across all providers, we observed that the higher the tumor stage, the higher the median number of recovered lymph nodes. In addition, we found that laparoscopic surgeries were associated with fewer recovered lymph nodes compared to open surgeries after adjusted for tumor stage.

More importantly, we found that higher lymph node recovery made a significant difference in patient survival. Five-year overall survival became optimal with an increase of $20 \%$ when the number of recovered lymph nodes were above the following thresholds: 15 lymph nodes for stage I and 25 lymph nodes for stages II and higher. Furthermore, increase in 5-year overall survival due to higher lymph 
node recovery was most profound among patients with localized tumors while increase in 5-year overall survival due to chemotherapy was most profound among patients with loco-regional tumors. These data suggest that control of microscopic disease through recovery of regional lymph nodes is more effective in localized tumors than locoregional tumors where cancer is likely spread beyond regional nodes, thus pointing to the importance of systemic therapy for loco-regional tumors.

Indeed, our study results confirmed that the combination of higher lymph node recovery and chemotherapy was associated with a significant increase in survival among patients with loco-regional tumors, from $15 \%$ among patients who had lower lymph node recovery and no chemotherapy to $45 \%$ among patients with higher lymph node recovery and chemotherapy. In reference to literature, the $45 \%$ survival rate observed in the best-performing subgroup in our cohort was higher than the 36-38\% survival rate reported for patients receiving surgery and chemotherapy in the European MAGIC and INT0116 trials $(2,3)$, but lower than the $78 \%$ survival rate among patients receiving D2 surgery and chemotherapy in the Asian CLASSIC trial (6). Reasons to explain these differences could include surgical quality and ethnic composition of our study cohort. Last but not least, $25 \%$ of the study patients received radiation therapy in addition to chemotherapy. Clinical trials directly comparing chemoradiotherapy to chemotherapy alone have been negative so far $(21,22)$ and our study confirmed that use of radiation therapy was not associated with improved survival. In summary, these findings highlight the importance of both adopting evidence-based therapies as standard practice and adhering to surgical guidelines that at least 15 lymph nodes be recovered during gastrectomy for any tumor stage.

A limitation of this study involves the retrospective nature of the cancer registry data, of which accuracy and completeness are variable. For example, we were not able to ascertain the reasons for inadequate lymph node recovery, which might include providers' lack of knowledge, lack of effort, lack of communication between surgeons and pathologists, and patient obesity. As another example, some patients were lost to follow-up and their deaths were not documented at the study institutions. Although we were able to obtain these patients' death events from the cancer registry database, we were not able to obtain the causes of their death. Despite these limitations, we have made extensive effort to improve the data accuracy and completeness by conducting our own chart reviews of the patients' health records to validate the clinical factors and outcomes. Another limitation is the non-randomization bias when comparing patients who did and did not receive chemotherapy; that is, patients who did not receive chemotherapy may have been less fit for therapy and thus more likely to have poorer survival outcomes irrespective of chemotherapy administration; to reduce this bias, we compared survival among patients of similar tumor stages and comorbidities. In addition, we were not able to evaluate differences amongst chemotherapy regimens and radiation doses due to lack of available data. However, chart reviews indicate that the most common regimens administered at our study sites consisted of fluoropyrimidine-platinum doublets. We would not expect any differences in survival among the chemotherapy regimens since the MAGIC and INT0116 trials of these chemotherapy regimens showed similar overall survival rates $(2,3)$ while the ALLIANCE trial showed that addition of more intensified chemotherapy regimens to chemoradiotherapy did not improve survival (23). Finally, we could not map our lymph node thresholds to the D2 lymphadenectomy protocol because we do not have detailed information about the recovered lymph node stations. However, studies have shown that the total number of recovered lymph nodes as a proxy for the extent of lymphadenectomy was a significant predictor of survival $(8,24,25)$. Despite these limitations, our study results were consistent with randomized controlled trials from literature on that greater lymph node recovery yielded superior survival benefits and that addition of radiation therapy to chemotherapy did not confer further improvement in survival. The strength of our study is that the data was derived from a contemporary patient cohort in multiple high-volume institutions, and thus might be more generalizable to current practices. Our next step is to develop and implement a provider activation program to promote adherence to evidence-based therapies and surgical guidelines for the treatment of gastric cancer as we believe that lack of adherence to clinical guidelines is an issue in the United States where gastric cancer is not a common disease.

\section{Acknowledgments}

Funding: This work was partially supported by grant UL1 TR001414 from the National Center for Advancing Translational Sciences, National Institutes of Health (NIH), through the Biostatistics, Epidemiology and Research Design Unit. The content is solely the responsibility of the authors and does not necessarily represent the official views 
of the NIH.

\section{Footnote}

Reporting Checklist: The authors present the study in accordance with the STROBE reporting checklist. Available at http://dx.doi.org/10.21037/jgo-20-212

Data Sharing Statement: Available at http://dx.doi. org/10.21037/jgo-20-212

Peer Review File: Available at http://dx.doi.org/10.21037/ jgo-20-212

Conflicts of Interest: All authors have completed the ICMJE uniform disclosure form (available at http://dx.doi. org/10.21037/jgo-20-212). Outside this submitted work, FD reports grants and personal fees from AZD, BMS, Taiho, Merck, Genentech, FMI, Amgen, Array, Deciphera, Eisai, Exelixis, Ipsen, QED, Signatera, and Sirtex. The other authors have no conflicts of interest to declare.

Ethical Statement: The authors are accountable for all aspects of the work in ensuring that questions related to the accuracy or integrity of any part of the work are appropriately investigated and resolved. The study was conducted in accordance with the Declaration of Helsinki (as revised in 2013). This study was approved by the Institutional Review Boards at the Universities of California in Davis, Irvine, Los Angeles, and San Francisco prior to the collection of data (UC Reliance No. 2843). Because of the retrospective nature of the research, the requirement for informed consent was waived.

Open Access Statement: This is an Open Access article distributed in accordance with the Creative Commons Attribution-NonCommercial-NoDerivs 4.0 International License (CC BY-NC-ND 4.0), which permits the noncommercial replication and distribution of the article with the strict proviso that no changes or edits are made and the original work is properly cited (including links to both the formal publication through the relevant DOI and the license). See: https://creativecommons.org/licenses/by-nc-nd/4.0/.

\section{References}

1. Ferlay J, Soerjomataram I, Ervik M, et al. Cancer Incidence and Mortality Worldwide: IARC CancerBase
No. 11. Lyon, France: International Agency for Research on Cancer, 2013. GLOBOCAN 2012 v1.0.

2. Macdonald JS, Smalley SR, Benedetti J, et al. Chemoradiotherapy after surgery compared with surgery alone for adenocarcinoma of the stomach or gastroesophageal junction. N Engl J Med 2001;345:725-30.

3. Cunningham D, Allum WH, Stenning SP, et al. Perioperative chemotherapy versus surgery alone for resectable gastroesophageal cancer. $\mathrm{N}$ Engl J Med 2006;355:11-20.

4. Ychou M, Boige V, Pignon JP, et al. Perioperative chemotherapy compared with surgery alone for resectable gastroesophageal adenocarcinoma: an FNCLCC and FFCD multicenter phase III trial. J Clin Oncol 2011;29:1715-21.

5. Al-Batran SE, Homann N, Pauligk C, et al. Perioperative chemotherapy with fluorouracil plus leucovorin, oxaliplatin, and docetaxel versus fluorouracil or capecitabine plus cisplatin and epirubicin for locally advanced, resectable gastric or gastro-oesophageal junction adenocarcinoma (FLOT4): a randomised, phase 2/3 trial. Lancet 2019;393:1948-57.

6. Bang YJ, Kim YW, Yang HK, et al. Adjuvant capecitabine and oxaliplatin for gastric cancer after D2 gastrectomy (CLASSIC): a phase 3 open-label, randomised controlled trial. Lancet 2012;379:315-21.

7. Sakuramoto S, Sasako M, Yamaguchi T, et al. Adjuvant chemotherapy for gastric cancer with S-1, an oral fluoropyrimidine. N Engl J Med 2007;357:1810-20. Erratum in: N Engl J Med. 2008 May 1;358(18):1977.

8. Karpeh MS, Leon L, Klimstra D, et al. Lymph node staging in gastric cancer: is location more important than Number? An analysis of 1,038 patients. Ann Surg 2000;232:362-71.

9. Schwarz RE, Smith DD. Clinical impact of lymphadenectomy extent in resectable gastric cancer of advanced stage. Ann Surg Oncol 2007;14:317-28.

10. Cuschieri A, Weeden S, Fielding J, et al. Patient survival after D1 and D2 resections for gastric cancer: long-term results of the MRC randomized surgical trial. Surgical Cooperative Group. Br J Cancer 1999;79:1522-30.

11. Hartgrink HH, Van de Velde CJ, Putter H, et al. Extended lymph-node dissection for gastric cancer: who may benefit? Final results of the randomized Dutch gastric cancer group trial. J Clin Oncol 2004;22:2069-77.

12. Songun I, Putter H, Kranenbarg EM, et al. Surgical treatment of gastric cancer: 15 -year follow-up results of 
the randomised nationwide Dutch D1D2 trial. Lancet Oncol 2010;11:439-49.

13. Degiuli M, Sasako M, Ponti A, et al. Survival results of a multicentre phase II study to evaluate D2 gastrectomy for gastric cancer. Br J Cancer 2004;90:1727-32.

14. Wu CW, Hsiung CA, Lo SS, et al. Nodal dissection for patients with gastric cancer: a randomised controlled trial. Lancet Oncol 2006;7:309-15.

15. Sasako M, Sano T, Yamamoto S, et al. D2 lymphadenectomy alone or with para-aortic nodal dissection for gastric cancer. $\mathrm{N}$ Engl J Med 2008;359:453-62.

16. Degiuli M, De Manzoni G, Di Leo A, et al. Gastric cancer: Current status of lymph node dissection. World J Gastroenterol 2016;22:2875-93.

17. The California Cancer Registry Data Dictionary. Available online: http://dd.ccr.ca.gov/. Last access on January 15, 2020.

18. Hamilton SR, Aaltonen LA. editors. Tumours of the Digestive System. In: World Health Organization Classification of Tumours: Pathology and Genetics. Lyon, France: IARC Press, 2000.

19. Kunz PL, Gubens M, Fisher GA, et al. Long-term survivors of gastric cancer: a California population-based study. J Clin Oncol 2012;30:3507-15.

20. The Surveillance, Epidemiology, and End Results (SEER)

Cite this article as: Hoang T, Dayyani F, Alfaro A, Huynh J, Ji J, Ko AH, Cho M, Hiyama D. Variability in multimodality treatment influences survival in non-metastatic gastric cancer patients. J Gastrointest Oncol 2020;11(5):952-963. doi: 10.21037/ jgo-20-212
Program. Available online: https://seer.cancer.gov/ statfacts/html/stomach.html

21. Park SH, Sohn TS, Lee J, et al. Phase III Trial to Compare Adjuvant Chemotherapy with Capecitabine and Cisplatin versus Concurrent Chemoradiotherapy in Gastric Cancer: Final Report of the Adjuvant Chemoradiotherapy in Stomach Tumors Trial, Including Survival and Subset Analyses. J Clin Oncol 2015;33:3130-6.

22. Cats A, Jansen EPM, van Grieken NCT, et al. Chemotherapy versus chemoradiotherapy after surgery and preoperative chemotherapy for resectable gastric cancer (CRITICS): an international, open-label, randomised phase 3 trial. Lancet Oncol 2018;19:616-28.

23. Fuchs CS, Niedzwiecki D, Mamon HJ, et al. Adjuvant Chemoradiotherapy With Epirubicin, Cisplatin, and Fluorouracil Compared with Adjuvant Chemoradiotherapy With Fluorouracil and Leucovorin After Curative Resection of Gastric Cancer: Results From CALGB 80101 (Alliance). J Clin Oncol 2017;35:3671-7.

24. Smith DD, Schwarz RR, Schwarz RE. Impact of total lymph node count on staging and survival after gastrectomy for gastric cancer: data from a large USpopulation database. J Clin Oncol 2005;23:7114-24.

25. Coburn NG, Swallow CJ, Kiss A, et al. Significant regional variation in adequacy of lymph node assessment and survival in gastric cancer. Cancer 2006;107:2143-51. 


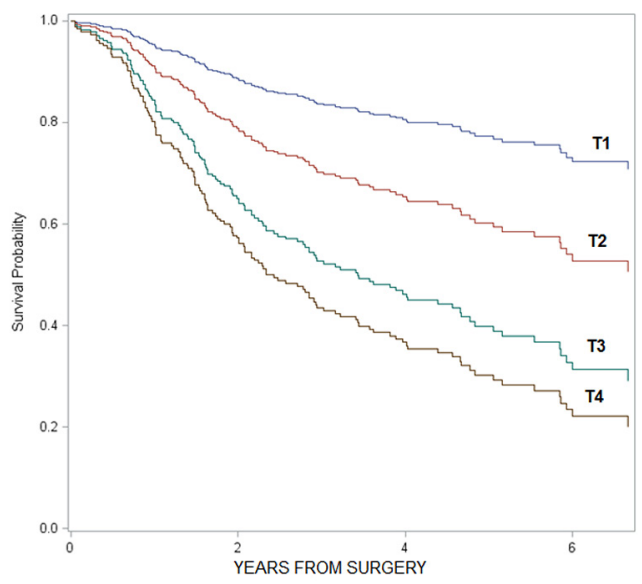

Figure S1 Comparison of overall survival among $\mathrm{T}$ stages.

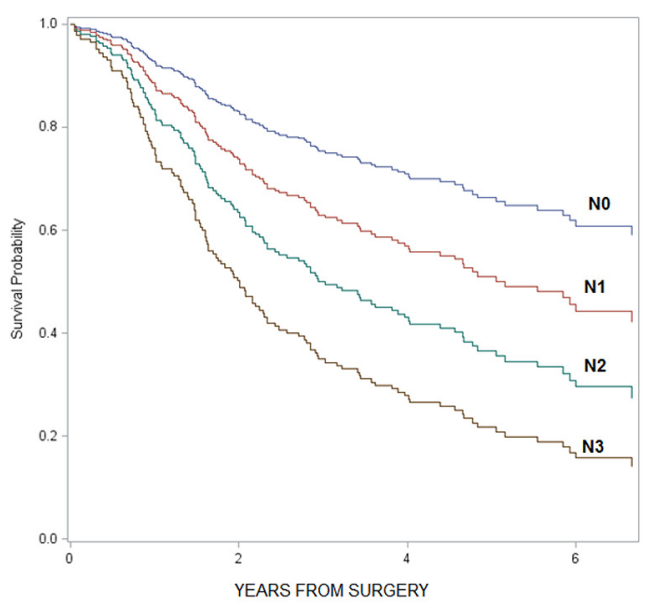

Figure S2 Comparison of overall survival among N stages. 
Table S1 Variability in multimodality treatments

\begin{tabular}{|c|c|c|c|}
\hline Treatments & Localized tumors $(n=169)$ & Loco-regional tumors $(n=157)$ & $P$ value \\
\hline 0 & 9.5 & 4.5 & \\
\hline $1-14$ & 33.1 & 21.7 & \\
\hline $15-24$ & 26.6 & 26.7 & \\
\hline Unknown/NA & 6.5 & 2.5 & \\
\hline Chemotherapy administration (\%) & & & $<0.0001$ \\
\hline Neoadjuvant alone & 19.5 & 19.7 & \\
\hline Adjuvant alone & 9.5 & 42.7 & \\
\hline Multimodality treatment (\%) & & & $<0.0001$ \\
\hline Surgery only & 63.9 & 22.9 & \\
\hline Surgery/chemotherapy & 20.1 & 42.0 & \\
\hline Surgery/chemotherapy/radiation & 16.0 & 35.1 & \\
\hline
\end{tabular}

Table S2 Comparison of comorbidity and weight loss among treatment groups

\begin{tabular}{|c|c|c|c|c|c|c|}
\hline Treatment groups & \multicolumn{3}{|c|}{ High comorbidity burden } & \multicolumn{3}{|c|}{ Weight loss at diagnosis } \\
\hline Localized tumors & & & 0.5722 & & & 0.8357 \\
\hline Higher lymph node recovery & 69 & 10.1 & & 69 & 27.5 & \\
\hline Lower lymph nodes recovery & 100 & 13.0 & & 100 & 29.0 & \\
\hline Higher lymph node recovery & & & 0.9080 & & & 0.5818 \\
\hline Chemotherapy & 62 & 8.1 & & 62 & 43.5 & \\
\hline No chemotherapy & 14 & 7.1 & & 14 & 35.7 & \\
\hline Lower lymph node recovery & & & 0.6318 & & & 0.1682 \\
\hline
\end{tabular}




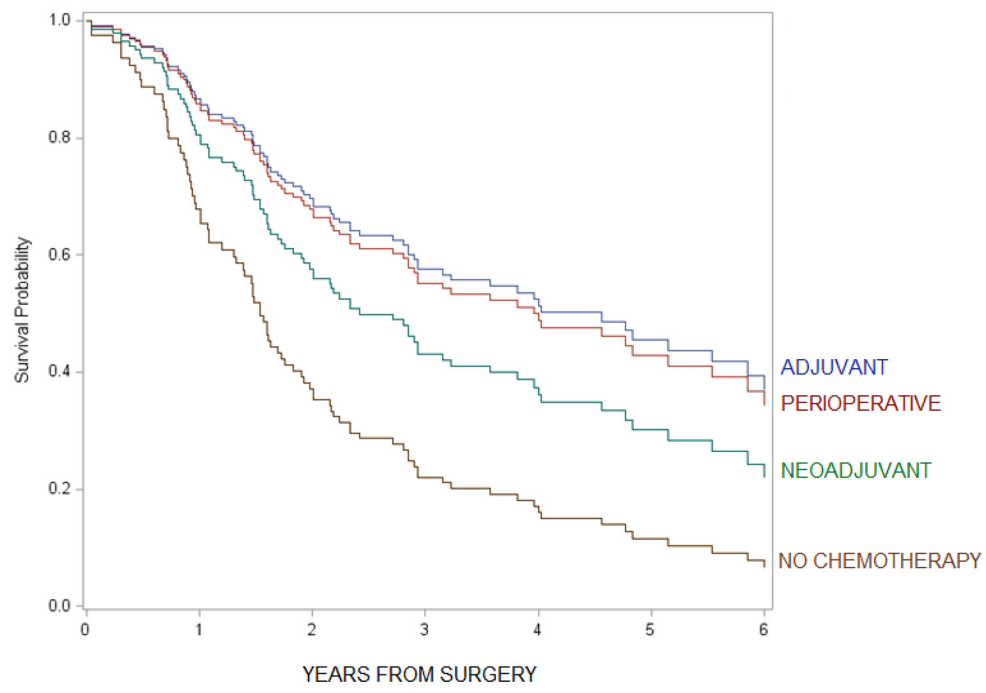

Figure S3 Comparison of overall survival among chemotherapy administrations in loco-regional tumors.

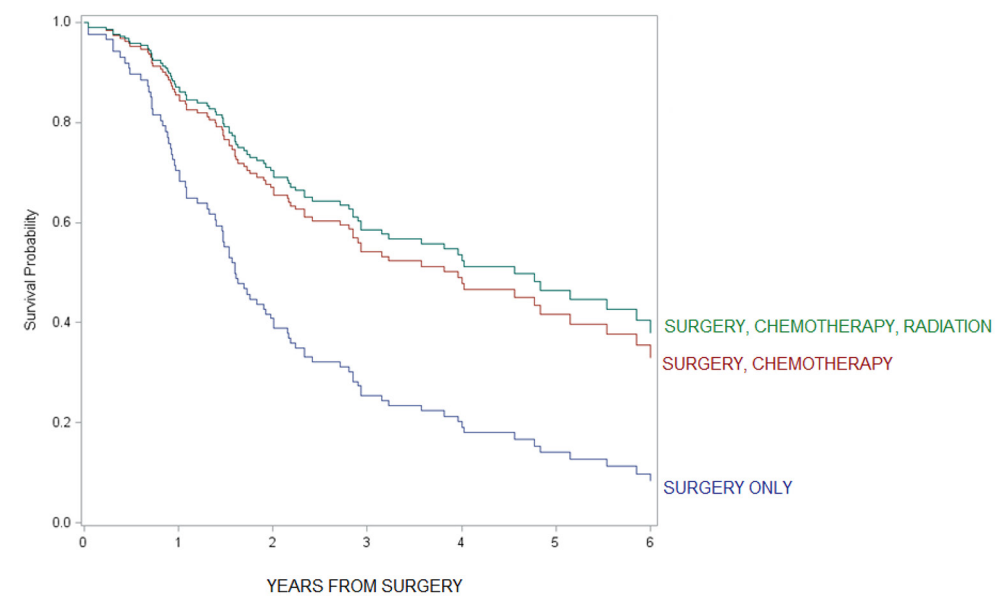

Figure S4 Comparison of overall survival among multimodality treatments in loco-regional tumors. 